\title{
Henry Shoshkes, un voyageur juif polonais entre les deux guerres
}

Henry Shoshkes, a Polish Jewish Traveler between the two World-Wars

\section{Judith Lindenberg}

\section{OpenEdition}

\section{Journals}

Édition électronique

URL : https://journals.openedition.org/recherchestravaux/860

DOI : 10.4000/recherchestravaux.860

ISSN : 1969-6434

Éditeur

UGA Éditions/Université Grenoble Alpes

Édition imprimée

Date de publication : 1 décembre 2016

Pagination : 109-118

ISBN : 978-2-84310-338-4

ISSN : 0151-1874

Référence électronique

Judith Lindenberg, "Henry Shoshkes, un voyageur juif polonais entre les deux querres », Recherches \& Travaux [En ligne], 89 | 2016, mis en ligne le 12 janvier 2017, consulté le 29 octobre 2021. URL : http:// journals.openedition.org/recherchestravaux/860 ; DOI : https://doi.org/10.4000/recherchestravaux. 860 
Judith Lindenberg

EHESS, Centre de recherches historiques

\section{Henry Shoshkes, un voyageur juif polonais entre les deux guerres}

Pour comprendre le parcours d'Henry (Khaim) Shoshkes et étudier ses écrits de reportage, il semble nécessaire de retracer son itinéraire biographique et de le replacer dans le contexte intellectuel et historique de son époque. Juif polonais yiddishophone dont l'essentiel de l'existence se déroula durant la première moitié du xx siècle, Henry Shoshkes (I89I-1964) est tombé dans l'oubli et le milieu dans lequel il évolua demeure mal connu, en Pologne comme ailleurs.

Né à Białystok dans une famille juive traditionnelle religieuse, Shoshkes a fait ses études secondaires dans un lycée russe ${ }^{\mathrm{I}}$ et a poursuivi ses études supérieures d'économie dans plusieurs universités en Russie et à l'étranger. En 19I4, il revient en Russie et travaille à la coopérative de crédit de Kharkov; il assiste donc à la Révolution et à la guerre civile jusqu'à son retour en Pologne en I92I. À Varsovie, il continue de travailler dans une banque coopérative; il effectue de nombreux voyages professionnels dans des contrées reculées de la Pologne, dont il tire des reportages pour la presse yiddishophone et polonophone. Entre les deux guerres, il est l'un des principaux responsables des mouvements de coopération internationaux, en travaillant notamment pour le Joint Distribution Committee et la HIAS (Hebrew Immigrant Aid Society). Le Joint Distribution Committee, communément appelé le Joint, basé à New York, est une organisation humanitaire non politique fondée en I9I4 pour aider les victimes de guerre² ${ }^{2}$ la HIAS avait été fondée originairement en i88I pour aider les Juifs à fuir les pogroms en Russie et dans l'est de l'Europe. Ces deux

I. À la fin du XVIII ${ }^{\text {e }}$ siècle, le pays était partagé entre l'Empire austro-hongrois, la Prusse et l'Empire russe; Białystok, comme toute la partie orientale, se trouvait sous domination russe.

2. À propos du Joint, voir G. Estraikh, R. Zalashik, "Taking and Giving : The Case of the JDC and OZE in Lithuania 1919-26", East European Jewish Affairs, 2009. 
organisations - qui existent toujours - ont donc eu pour mission d'aider les Juifs face aux fléaux qui s'abattent sur eux à l'aube du xx ${ }^{e}$ siècle. Dans le cadre de ces activités, Shoshkes effectue également de nombreux voyages, à l'étranger, sur lesquels il écrit également des reportages pour la presse.

Au début de la Deuxième Guerre mondiale, il fait brièvement partie du Conseil juif (Judenrat) de Varsovie. Grâce à ses relations, il peut se procurer de faux papiers et s'échapper de Pologne, avec sa femme et son fils, dès 1939. Après un détour par l'Italie, il parvient à New York à la fin de l'année 1939, où il vit jusqu’à sa mort en 1964 .

En Shoshkes, à l'image de nombreuses personnalités de sa génération, coexistaient les deux facettes, juive et polonaise, de son identité : il connaissait toute la culture polonaise de son temps, ainsi que la culture juive sécularisée, elle-même fortement nourrie de culture polonaise. Son sionisme militant, son travail dans les organisations internationales était le signe de son implication politique dans l'émancipation des Juifs, que ce soit à travers l'aide américaine ou l'émigration en Palestine.

Cette double identité s'accompagnait la plupart du temps chez les intellectuels de la génération de Shoshkes d'un bilinguisme polonais et yiddish voire d'un trilinguisme avec l'hébreu. Cependant un nombre relativement important d'intellectuels juifs polonais étaient surtout ou exclusivement polonophones car complètement assimilés; écrivant leur œuvre dans cette langue, ils ont pu avoir une postérité, même tardive, et être intégrés dans le patrimoine polonais. On pourrait citer à cet égard l'écrivain et pédagogue Janusz Korczak (I878-1942), le poète Julian Tuwim (I894-1953) ou l'écrivain et dessinateur Bruno Schulz (I892-1942). Le choix du yiddish comme langue d'écriture, un choix identitaire à l'origine, a été opéré par des intellectuels dans leur grande majorité polonophones, mais voulant promouvoir cette langue qui leur était propre et qui avait un ancrage populaire. En effet, le yiddish était devenu, depuis le début $\mathrm{du} \mathrm{xx}^{\mathrm{e}}$ siècle, après une phase de maturation et d'éclosion de la littérature et des mouvements identitaires et politiques au XIX ${ }^{\mathrm{e}}$ siècle - et surtout depuis la conférence de Czernowitz en 1908, qui l'a imposé comme langue d'une nation juive ashkénaze en construction - le socle de la culture littéraire et politique des Juifs polonais ${ }^{3}$. Ce moment a coïncidé avec le début de la sécularisation de la jeune génération juive polonaise, qui avait à l'instar de Shoshkes délaissé, au début du siècle, le cadre de vie religieux pour une culture laïque. Ce choix a rendu l'œuvre de tous ces auteurs, du fait de la

3. Voir à ce sujet I. N. Gottesman, Defining the Yiddish Nation: The Jewish Folklorists of Poland, Wayne State University Press, 2000; Weiser, Kalman, Jewish People, Yiddish Nation, Toronto, Toronto Press University, 201 . 
destinée de la langue yiddish après le génocide 4 , inaccessible pour les lecteurs d'aujourd'hui après une période d'effervescence culturelle, aussi bien dans le domaine de l'art, de la littérature et du théâtre que dans celui des sciences sociales ${ }^{5}$, avec beaucoup moins de clivages entre ces différents domaines que nous n'en connaissons aujourd'hui. De ce point de vue, le choix, opéré par Shoshkes, de conjuguer ses activités sociales et politiques avec ses activités d'écrivain et de journaliste est représentatif de l'intelligentsia juive polonaise de l'entre-deux-guerres. Par sa participation à des organisations humanitaires, il appartient à un cercle plus restreint à l'intérieur de cette intelligentsia, qui était déjà internationalisée tout en conservant son centre en Pologne.

Mais c'est surtout pour ses récits de voyage que Shoshkes était connu, et cela constitue une particularité. Leur publication dans la presse juive yiddishophone et polonophone - Haynt [Aujourd'hui] et Nasz Przeglad [Notre revue] notamment ${ }^{6}-$ montre chez l'auteur une égale maîtrise et un goût partagé des deux langues. Cependant seuls ses articles en yiddish ont fait l'objet de publications en volume, ce qui pose des questions auxquelles la suite de cet article apportera une réponse partielle.

Tout en se rattachant à une pratique d'écriture spécifiquement polonaise, peu pratiquée par les écrivains juifs, Shoshkes a manifesté dans ses récits de voyage, parallèlement au goût de la découverte et de l'altérité propre à tous les voyageurs, une préoccupation spécifique pour le sort des Juifs dans le monde. Surtout, il y a développé une manière de raconter tributaire à la fois des savoirs et des pratiques littéraires juifs et polonais de son temps, réalisant ainsi une synthèse de ses deux identités. Shoshkes a écrit de nombreux récits de ses voyages dans le monde entier : la liste de ses ouvrages ressemble à une visite méthodique de tous les continents. En témoignent les titres de ses ouvrages publiés de 1930 à 1954 : Mein reize arum der velt [Mes voyages autour du monde $]^{7}$, Durkh unbakante lender $[\grave{A} \text { travers des pays inconnus }]^{8}$ (I954), dans

4. R. Ertel, Dans la langue de personne, Paris, Seuil, 1993.

5. Cette période était au cœur de l'exposition Futur antérieur, conçue par Nathalie HazanBrunet et Hillel Kazovsky, qui s'est tenue au musée d'Art et d'histoire du judaïsme en 2009. Catalogue Futur antérieur. L'Avant-garde et le livre yiddish, Paris, Flammarion, 2009.

6. Le premier était l'un des principaux quotidiens de la presse yiddish, de tendance sioniste, publié à Varsovie; le second, son équivalent polonophone, était également sioniste et publié à Varsovie, lancé et dirigé par Janusz Korczak entre 1926 et 1930. Il présentait par ailleurs un supplément pour enfants écrit par les enfants. La participation à des journaux dans les deux langues souligne la double identité de l'auteur. Voir E. Prokop-Janiec, Polish-Jewish Literature in the Interwar Years, New York, Syracuse University Press, 2003.

7. H. Shoshkes, Mein reize arum der velt [Mes voyages autour du monde], Buenos Aires, Unzer bukh, I95I.

8. H. Shoshkes, Durkh unbakante lender [À travers des pays inconnus], Rio de Janeiro, Monte Scopus, I954. 
lesquels les titres de chapitres évoquent des voyages en Afrique, en Amérique du Nord et du Sud, etc. Entretemps, il a écrit également sur l'extermination des Juifs de Pologne, Bleter fun a geto togbukh [Feuillets pour un journal du ghetto] publié à New York en $1943^{\circ}$, puis, à Buenos Aires, dans la plus grande collection en yiddish d'après-guerre ${ }^{\mathrm{IO}}$, Poyln 1946 [Pologne 1946 $]^{\mathrm{II}}$ - sur laquelle on reviendra - puis A velt vos iz farbay... Kapitel zikhoynes [Un monde qui n'est plus... Chapitres de Mémoires $]^{12}$.

L'ouvrage Lender un shtet : reize eindrukn mit bilder [Pays et villes : impressions de voyage illustrées] publié en 1930 à Vilnius ${ }^{13}$ est également consacré à des zones du monde diverses et, pour certaines, traitées dans d'autres ouvrages de Shoshkes : l'Italie, Égypte, la Palestine - appelée par les sionistes «Eretz Israël»-, l'Afrique du Nord, l'Espagne, les Balkans, la Syrie. Cet ouvrage, le premier publié par l'auteur, a la particularité de comporter une section qui s'intitule Poylen (Pologne en yiddish). Une étude de l'ensemble de ses reportages reste à faire tant son ouvre demeure, comme celle de la quasi-totalité de ses compatriotes Juifs polonais yiddishophones, méconnue; mais c'est à cet aspect, en apparence mineur, qu'on va s'intéresser, en le reliant à l'émergence de l'ethnographie judéo-polonaise dans la première moitié $\mathrm{du} \mathrm{xx}^{\mathrm{e}}$ siècle. Cette ethnographie avait, en effet, remis au goût du jour chez les intellectuels le voyage "près de chez soi», sans pour autant que cette tendance fasse éclore un genre littéraire.

Premier élément à souligner : Shoshkes n'a pas constitué de recueil de ses voyages en Pologne. Il en a inclus une partie ${ }^{14}$ dans un ouvrage contenant des récits de voyage dans le monde entier. Ce choix éditorial encourage à nuancer l'opposition entre le familier et l'étranger, si l'on se replace dans le contexte de la Pologne des années 1920, pays jeune et tout juste réunifié après son

9. H. Shoshkes, Bleter fun a geto togbukh [Feuillets pour un journal du ghetto], New York, Glanz, I943.

Io. Sur cette collection, voir J. Schwarz, "A Library of Hope and Destruction : The Yiddish Book Series "Dos Poylishe Yidntum" ("Polish Jewry") I946-1966», Polin, n 20, 2008 et J. Lindenberg, "Dos poylishe yidntum : histoire et mémoire d'une collection au lendemain de la Catastrophe» (1946-1966)", Plurielles, n' 19, 2015.

II. H. Shoshkes, Poyln 1946. Eindruk fun a reize [Pologne 1946. Impressions de voyage], Buenos Aires, Union des Juifs polonais en Argentine, 1946.

I2. H. Shoshkes, A velt vos iz farbay... Kapitel zikhoynes [Un monde qui n'est plus... Chapitres de Mémoires], Buenos Aires, Union des Juifs Polonais en Argentine, I949.

I3. L'actuelle capitale de Lituanie, alors appelée Wilno en polonais (Vilna en russe, Vilne en yiddish) après avoir appartenu à l'Empire russe, connut quelques années de turbulences après 19I8, et fit partie de la Pologne à partir de 1922.

I4. Tout laisse à penser qu'il a publié dans la presse de nombreux autres reportages sur ses voyages en Pologne; l'étude, facilitée désormais par la numérisation d'une grande partie de la presse yiddish, en reste encore à faire. 
écartèlement pendant plus d'un siècle entre trois empires, qui ont imprimé à chacune de ses parties des identités propres. Sur les dix-neuf chapitres que compte cette partie, plusieurs sont consacrés au shtetl, nom yiddish donné aux petites villes et aux villages de Pologne comptant une forte population juive, ou à la partie de ces villes et villages habités par les Juifs. Ce terme a fini par acquérir une résonance folklorique, pour devenir un condensé de la vie rurale juive polonaise d'avant la Shoah. Il s'agit «non seulement d'un lieu habité par des semblables, mais aussi d'une structure économique et sociale particulière, d'un espace juif", tel que l'a défini Rachel Ertel dans son ouvrage pionnier Le shtetl. La bourgade juive de Pologne ${ }^{15}$. Le shtetl a été constitué en espace mythique après la Shoah; mais déjà avant la Seconde Guerre mondiale, du fait de l'urbanisation galopante pendant l'entre-deux-guerres notamment pour les populations juives en proie à une forte paupérisation, il est peu à peu devenu un espace relégué, en retrait des grandes agglomérations, un espace perçu comme plus authentique et détenteur des valeurs ancestrales du judaïsme, et, pour ces raisons, fortement prisé par les ethnographes. Dans cette partie du livre, Shoshkes se fait le porte-parole direct d'un tel état des choses.

Au cours de son périple, Shoshkes rend compte de la présence simultanée de la pauvreté et de la soif de culture dans les contrées reculées de la Pologne qu'il traverse. Dans un chapitre intitulé "Shtetl dans la boue», il montre comment, dans des régions où, dit-il, ne passent ni trains ni bus, «la poste apporte les journaux de la capitale, un livre tout juste publiér ${ }^{16} »$. Cette circulation des nouveautés entraîne une grande agitation parmi la jeunesse : "on discute dans les écoles, dans les bibliothèques, on discute de tous les feuilletons ou de la nouvelle œuvre d'un jeune écrivain yiddish". Pourtant, rien n'est fait pour encourager une telle soif de culture car - le voyageur le répète comme un leitmotiv - «le shtetl est pauvre». À cause de cela, «il n'y a pas de professeur, pas de représentant des partis politiques» mais, dit-il, «on vit là, avec en son cœur le désir du grand monde ${ }^{17} »$. Ces mots rendent bien compte de l'opposition, dans le monde judéo-polonais, entre la vitalité de la culture, et la réalité économique très dure à laquelle les habitants des shtetlekh étaient confrontés. Cette situation recoupe plus largement les oppositions - non exactement superposables - qui séparaient le monde rural et le monde urbain, le mode de vie religieux traditionnel et le mode de vie sécularisé, le peuple et les intellectuels, et interroge en creux le rapport même de Shoshkes à son sujet.

I5. R. Ertel, Le Shtetl. La Bourgade juive de Pologne, Paris, Payot, I983.

I6. R. Shoshkes, "Shtetl in blotes» ["Shtetl dans la boue»], dans Pays et villes autour $d u$ monde, Vilnius, Klezkin, p. 27I. Les traductions sont de J. Lindenberg.

I7. Ibid. 
En effet, si, dans ce chapitre, Shoshkes ne nomme pas le shtetl en question, le faisant ainsi devenir un shtetl par antonomase, on comprend à travers quelques allusions qu'on se trouve dans la région de Białystok, qui est la sienne. Cependant la distance ici n'est pas géographique mais sociale : pour l'écrivain né dans un milieu certes populaire et traditionnel mais urbain, qui s'est élevé socialement, la réalité du shtetl est lointaine. Ce geste de l'écrivain retournant à sa terre d'origine se rattache à une figure très importante dans la culture judéo-polonaise : celle d'Isaac Leibush Peretz. Couramment désigné comme l'un des "pères de la littérature yiddish " et connu pour ses nouvelles et ses romans, Peretz a, en effet, fait ses débuts de romancier en rédigeant ce qui devait être un travail alimentaire : le rapport d'une enquête ethnographique, qui sera finalement publiée sous le titre de Tableaux d'un voyage en province ${ }^{18}$. Cette enquête, menée en I890, avait été financée par un riche philanthrope polonais, Jan Bloch. C'est à la même période que Simon Doubnov, historien juif russe, a contribué à la création d'une commission historico-ethnographique dont le but était d'attester la présence juive dans l'empire russe ${ }^{\mathrm{r}}$. Bloch, un important banquier, entrepreneur et philanthrope qui avait été influencé par les idées de Doubnov a lancé cette enquête qui s'est déroulée dans la région de Zamość dont Peretz était lui-même originaire; Peretz, le futur écrivain, qui vivait depuis longtemps à Varsovie, a découvert à cette occasion le monde des petites gens de sa région natale, et a été profondément frappé par leur misère. Ce qui devait être une enquête statistique s'est transformé en description sociale et psychologique des personnes rencontrées, de leur façon d'être et de parler. Ces «tableaux» ont constitué la matrice de son œuvre littéraire à venir. De plus, le choix de la langue yiddish n'avait à l'époque rien d'évident : Peretz avait été, à l'origine, plutôt hostile au yiddish comme langue d'écriture; ce n'est que deux ans auparavant, en I888, qu'il avait publié son célèbre premier poème, Monish $^{20}$, et contribué à faire du yiddish une langue littéraire. De plus, la référence implicite à Heinrich Heine et à ses Reisebilder [Tableaux de voyage], invite à sortir du monde judéo-polonais pour replacer cet écrit de jeunesse

I8. I. L. Peretz, Bilder fun a provinz reize [Tableaux d'un voyage en province]. Cet écrit a été traduit en français sous le titre Les Oubliés du shtetl (éd. et trad. N., M. Weinstock, Paris, Plon, coll. «Terre humaine», 2007); il comporte, outre le texte de Peretz, de très nombreuses annexes qui contextualisent cet écrit. Le passage du titre original au titre français est la marque, à travers un geste éditorial, d'un transfert culturel qui donne à lire cet ouvrage à la fois dans le contexte de son temps et à travers le prisme rétrospectif de la vision d'un monde disparu.

19. Voir la première partie de l'ouvrage de L. Jockusch, Collect and record!', Oxford, Oxford University Press, 2012.

20. Connu sous le titre La Ballade de Monish ou Monish, du nom du jeune garçon qui en est le protagoniste, ce long poème a été publié pour la première fois en I888, puis en I892 et repris en recueil en I9I4. I. L. Peretz, Lider un baladn [Poèmes et ballades], Vilnius, Kletskin, I925. 
de Peretz dans le contexte plus large du monde juif occidental, dans lequel la culture judéo-allemande exerçait une forte influence. Dans les origines du reportage, contextes savants et littéraires se superposent, au croisement des différentes aires culturelles de l'Europe orientale.

Shoshkes a certainement pratiqué le genre du récit de voyage, très ancré dans la tradition polonaise, avec ses voyages en Pologne; mais il s'inscrit également dans la veine peretzienne, par sa manière d'appréhender et de mettre à distance son propre monde, par l'ethnographie, par son intérêt pour les Juifs vivant "près de chez lui » et appartenant à un autre milieu social, par sa façon de décrire les personnes et leurs lieux de vie. Cependant, entre la fin du XIX ${ }^{e}$ siècle et l'entre-deux-guerres, l'ethnographie a changé de statut. L'intelligentsia judéo-polonaise se l'est appropriée et l'a transformée en outil pour appréhender le peuple juif. À ce titre, la présence, au milieu d'un chapitre d'une photographie reproduite en pleine page et sur laquelle on voit une place de marché très animée, participe de cette démarche. La légende indique "Au marché de Białystok», avec, en dessous, la mention "tiré d'un album de Katcyzne». Voilà qui nous rattache aussi directement aux travaux ethnographiques de cette époque, car Alter Katcyzne (I885-I94I), qui était à la fois un écrivain et un photographe très important de l'entre-deux-guerres, a témoigné, surtout grâce à ses photographies, de la vie quotidienne des Juifs polonais de l'époque, surtout les plus pauvres ${ }^{21}$. Il était proche de Peretz et surtout d'An Ski, le pionnier, avec Simon Doubnov, des enquêtes ethnographiques en Pologne ${ }^{22}$. La photographie en question n'est qu'indirectement liée au sujet du chapitre qui traite bien de cette région, mais pas précisément de cette ville ni de son marché. Shoshkes semble ainsi vouloir enrichir son récit de voyage par une illustration qui ne vaut pas seulement pour son contenu, mais pour son origine, se plaçant dans le courant des travaux des ethnographes de son temps. Le fait qu'il s'agisse d'une photographie déjà publiée, puisque tirée d'un album, renforce cette impression. L'ensemble de l'ouvrage comporte plusieurs photographies, mais celle-ci est la seule de la partie polonaise. Certaines photographies, très belles, révèlent une véritable intention anthropologique; d'autres illustrent la description de lieux exotiques, comme l'Algérie. La photo du marché est la seule qui comporte l'indication de sa provenance.

2I. Alter Kacyzne a bénéficié, après-guerre, d'une réception relativement importante pour son travail de photographe. Voir à ce propos les rééditions posthumes de son album de photographies : A. Kacyzne, Poyln, Jewish life in the old country, New York, YIVO, I965, rééd. New York, Metropolitan Books, I999.

22. Voir N. Deutsch, The Worlds of S. An-Sky, The Jewish Dark Continent, Cambridge, Harvard University Press, 201 . 
Tout se passe comme si Shoshkes écrivait sur la Pologne en mettant en œuvre son savoir d'ethnographe. Ainsi, dans le chapitre intitulé Galizie [Galicie], il marque d'emblée une grande distance avec son sujet, soulignant les différences entre cette région et ce qu'il désigne comme "chez nous». Il écrit à ce propos : «je n'ai pas étudié l'ethnographie juive de la Galicie ${ }^{23} »$. Outre qu'elle atteste le rôle joué à l'époque par l'ethnographie dans la culture judéo-polonaise, cette remarque souligne les différences régionales marquées, explicables par l'histoire du pays. Shoshkes distingue clairement la Petite Pologne qui, au début des partages, à la fin du XVIII ${ }^{\mathrm{e}}$ siècle, avait été rattachée à l'Autriche et à la Prusse, de ce qu'il désigne comme "bei unz» [chez nous], c'est-à-dire la Pologne du Congrès, placée sous la tutelle russe entre I8I5 et I868, date à laquelle elle est annexée par la Russie sous le nom de Pays de la Vistule. La partition de la Pologne dans l'entre-deux-guerres contribue à forger ce regard mi-régionaliste, mi-impérial, qui fait que Shoshkes se sent plus proche de sa contrée d'origine que de la Pologne en tant qu'entité nationale. Plusieurs éléments dénotent l'empreinte d'une culture juive russe dans le regard porté par Shoshkes : l'ethnographie judéo-polonaise est née en Russie, de même que la HIAS, organisation également née en Russie en réponse à la situation spécifique vécue par les Juifs de la «zone de résidence $»^{24}$. Ainsi, dans le récit du grand voyageur Shoshkes dans son propre pays, les différents types de distances (sociale, épistémologique, géographique) sont le signe d'un regard construit et critique. Le voyage près de chez soi permet à un Juif polonais des années 1930 d'interroger la dimension composite de son identité et de son pays.

Plusieurs aspects importants se dégagent aujourd'hui des reportages de Shoshkes. Dans le contexte de leur époque, ils apparaissent comme un témoignage aussi bien sur la vie juive de la Pologne de l'entre-deux-guerres que sur l'identité de leur auteur et sur sa manière de décrypter son propre monde. Valable pour tout reportage, ce constat permet de réinsérer Shoshkes dans la tradition polonaise, tout en montrant comment ce dernier a adapté la forme à ses préoccupations et à son regard spécifique de Juif polonais. La suite de l'histoire - le génocide des Juifs par les nazis - confère à ces reportages une valeur particulière. La constitution postérieure d'un monde du shtetl figé et idéalisé par la nostalgie, perçu à travers l'ombre portée de sa destruction, en rend d'autant plus précieuses les représentations à l'époque où il était en prise avec des problématiques de son temps.

23. H. Shoshkes, "Galicie», dans Pays et villes autour du monde, ouvr. cité, p. 3II.

24. La zone de résidence avait été créée par Catherine II en I79I, suite à l'annexion d'une partie de la Pologne, pour y regrouper tous les Juifs de Russie. Elle a été maintenue jusqu'en I9I7. 
Dans cette perspective, la suite de l'histoire personnelle de Shoshkes et de son œuvre, apporte, elle aussi, un éclairage intéressant sur la production littéraire de l'entre-deux-guerres. Shoshkes fait partie du faible pourcentage de Juifs $-90 \%$ des trois millions de Juifs Polonais disparaissent pendant le génocide - qui réussirent à s'échapper de Pologne et à éviter l'extermination. Grâce à leurs contacts en dehors de la Pologne, les membres des organisations internationales ont eu plus de facilité à fuir quand l'étau nazi s'est resserrées; ils ont donc été proportionnellement plus nombreux parmi l'intelligentsia juive survivante après le génocide, dont Shoshkes continuera à être un membre actif. Tout se passe comme si son mode de vie nomade et cosmopolite de l'entre-deux-guerres, alors atypique, préfigurait la figure du Juif survivant dans sa dimension transnationale; de plus, l'émigration massive des Juifs polonais survivants après le génocide contribuera à la constitution d'un réseau mondial de la judéité polonaise jusque dans les années soixante, animé par la circulation des personnes, des livres et des idées ${ }^{26}$. Au lendemain de la guerre, en 1946, Shoshkes a effectué un voyage en Pologne pour le compte du Jewish Labor Committee afin de rendre compte de l'état du pays, et du secours à porter aux Juifs, au lendemain du génocide, qu'on n'appelait pas encore Shoah mais khurbn en yiddish - Catastrophe. Il a fait en partie ce voyage accompagné d'un autre Juif polonais, le secrétaire général du Jewish Labor Committee, Yankev Pat, qui venait comme lui de Białystok et qui, comme lui, menait en parallèle une carrière d'écrivain. Il en a tiré un ouvrage constitué de reportages en différents lieux de la Pologne, Poyln 1946. Eindruk fun a reize [Pologne 1946. Impressions de voyages], publié la même année dans la plus grande collection d'ouvrages en yiddish de l'après-guerre, «Dos poylishe yidntum» [La judéité polonaise], basée à Buenos Aires ${ }^{27}$. Â exactement vingt ans de distance - la plupart des chapitres du voyage en Pologne de l'entre-deux-guerres sont datés de 1926 - Shoshkes, le reporter professionnel d'un pays qui venait de devenir le sien est devenu par l'ironie de l'Histoire reporter d'un pays qui n'était désormais plus le sien. C'est, comme il l'explique dans le livre, au cours de ce périple qu'il a compris que la Pologne ne pouvait plus être son pays, car la vie n'y était plus possible

25. Un télégramme de la Jewish Telegraphic Agency daté du 25 octobre 1949, à Londres, relate que «l'information a été reçue stipulant que le Dr. Henry Shoshkes, leader sioniste et dirigeant du système de banque coopérative en Pologne, resté avec sa famille à Varsovie, est sauf [...]. Il est le représentant de la Fédération à Varsovie, directeur de la banque centrale coopérative dans l'ancienne Varsovie et journaliste" (voir <http:/www.jta.org/1939/Io/25/ archive/henry-shoshkes-cooperative-bank-expert-reported-safe-in-warsaw>). Ce télégramme illustre bien l'insertion de Shoshkes dans un réseau transnational.

26. Sur la reconstruction de la culture yiddish en exil dans l'après-guerre, voir J. Schwarz, Survivors and Exile, Détroit, Wayne State University Press, 2015.

27. H. Shoshkes, Poyln 1946, ouvr. cité. 
pour les Juifs, en raison notamment de la résurgence de l'antisémitisme ${ }^{28}$. Si la forme demeure celle du reportage - le sous-titre vient le souligner - il ne s'agit plus du même type de voyage. Shoshkes a ainsi traversé le siècle, survivant à la disparition de la Pologne juive, dont il prolonge le souvenir - la plupart de ses reportages ayant été publiés après la guerre.

28. La période suivant la Libération fut marquée notamment par le pogrom de Kielce, au cours duquel environ quarante Juifs furent tués le 4 juillet 1946. Cet événement décida une grande partie des survivants à émigrer. La Pologne comptait 243 ooo Juifs en juillet 1946 (contre 74 000 Juifs en I945, l'accroissement s'expliquant par le retour des Juifs d'URSS), mais en I947, leur nombre était retombé à 1oo ooo. Voir J. Gross, La Peur. L'Antisémitisme en Pologne après Auschwitz, trad. de l'américain J. P. Ricard et du polonais X. Chantry, Paris, Calmann-Lévy, 2010 (l'ouvrage à été publié d'abord aux États-Unis et en américain en 2006). 